\title{
A novel integrated model for assessing landslide susceptibility mapping using CHAID and AHP pair-wise comparison
}

\begin{abstract}
This article uses an integrated methodology based on a chi-squared automatic interaction detection (CHAID) model combined with analytic hierarchy process (AHP) for pair-wise comparison to assess medium-scale landslide susceptibility in a catchment in the Inje region of South Korea. An inventory of 3596 landslide locations was collected using remote sensing, and a random sample comprising $30 \%$ of these was used to validate the model. The remaining portion $(70 \%)$ was processed by the nearest-neighbour index (NNI) technique and used for extracting the cluster patterns at each location. These data were used for model training purposes. Ten landslide-conditioning factors (independent variables) representing four main domains, namely (1) topology, (2) geology, (3) hydrology, and (4) land cover, were used to produce two landslide-susceptibility maps. The first landslide-susceptibility map (LSM1) was produced by overlaying the terminal nodes of the CHAID result tree. The second landslide-susceptibility map (LSM2) was produced using the overlay result of AHP pair-wise comparisons of CHAID terminal nodes. The prediction rate curve results were better with LSM2 (area under the prediction curve $($ AUC) $=0.80$ ) than with LSM1 (AUC = 0.76). The results confirmed that the integrated hybrid model has superior prediction performance and reliability, and it is recommended for future use in medium-scale landslidesusceptibility mapping.
\end{abstract}

Keyword: Landslide-susceptibility maps; Analytic hierarchy process (AHP); Chi-squared automatic interaction detection (CHAID) 